\title{
To the question about the prospects to the use of trigeneration energy sources based on hydropower
}

\begin{abstract}
This article discusses at promising directions of researches in the fields of energy saving and the creation of effective ecologically clean energy sources. To introduce such new technologies into the real energy sector, the features of their technical implementation are indicated. These directions of researches are related to modern depletion problems of the world's fuel resources and tightening to environmental safety requirements.
\end{abstract}

Keywords: fuel and energy sector; energy saving, energy efficiency, combined power supply, environmental friendliness, biomimicry, smartgrids, plug \& play, RES, RF, HPPs
Volume 5 Issue I - 2018

\author{
Alexander V Volkov, Artem V Ryzhenkov, \\ Alexander G Parygin, Andrew V Naumov, \\ Alexey A Druzhinin, Alexander A \\ Vikhylantsev, Marat R Dasaev \\ National Research University, Russia
}

\begin{abstract}
Correspondence: Alexander V.Volkov, National Research University “Moscow Power Engineering Institute”, I4, Krasnokazarmennaya st., Moscow, I I I250, Russian Federation, Russia, Email volkovav@mpei.ru
\end{abstract}

Received: November 16, 2017 | Published: February 06, 2018

\section{Introduction}

At present, the Russian Federation and many other countries of the world are in the scientific and technical search for ways to create alternative highly efficient energy supply systems. This is due to the need for a gradual transition from traditional methods of generating electricity to safer and environmentally friendly technologies.

One of the main aspirations today is to reduce the environmental burden on the environment ${ }^{1}$ according to Russian ${ }^{2}$ and European ${ }^{3}$ standards of quality of energy produced. Therefore, the urgent task is to develop the existing and new hydropower potential familiarization. It is the most stable energy resource from the class of renewable energy sources (RES). Its use is important, because there are many developed countries of the European continent (Czech Republic, Austria, for example) which are close in the level of hydro resources and features of the terrain territory unto the Russian Federation (RF).

The experience of the world's energy industry was confronted with the problem of "saturating" a greater share of hydro resources with large generating capacities. Today, it brings forth a global trend of small and micro-scale generating capacities development. It is also necessary for the creation of highly integrated intelligent systemforming and distribution electric networks of a new generation (Smart Grids). And the third need is maintenance of upgrading and improving the reliability of the commissioned power plants, including large ones.

Even in the Russian Federation, which continues to be the natural resources base for many countries in the world today the scientific

${ }^{1}$ Decree of the Russian Federation President of the of August 1, 2015 No. 392

"About carrying out in the Russian Federation the Year of specially protected natural territories"

${ }^{2}$ GOST 32144-2013 "Electric energy. Electromagnetic compatibility of technical equipment. Power quality limits in the public power supply systems"

${ }^{3}$ EN 50160 "Voltage Characteristics of Public Distribution Systems" and technical problems solution in the development of energy-saving technologies and clean energy new methods are among the first. The power equipment depreciation measure of the Russian hydraulic power stations (HPPs) majority exceeds $40 \%$. This indicator directly indicates that not only small, but also large hydropower engineering should be subject to scientific and technological development. Its energy facilities are created by well thought out design algorithms. One of the relevant and effective directions in this regard may be the study of nature-friendly technologies (biomimicry). This scientific approach ensures the transfer of new positive hydrodynamic properties to the working bodies of hydropower equipment.

For example, the transfer of effective hydrodynamic properties that are used by the ichthyofauna members (researched also by the American Institute of Physics) when moving in an aqueous medium ${ }^{1}$ (Figure 1) or features of the birds body structure (researched also by the Aachen University) in order to reduce energy consumption in flight and for hunting ${ }^{2}$ (Figure 2).

It should be noted that some biomimicry regularities have already been studied by the NRU "MPEI" scientific group. For example, it has been established that the use of hetero and homogeneous configurations of the turbine impeller blade system contributes to the effective working area expansion by $15-20 \%$. Use of hydrophobic effect of the lotus leaf structure when it is used on the hydropower equipment working bodies reduces the energy losses in the flow part by $3-4 \%$ (Figure 3). Together with the questions of biomimicry in these studies, ways of improving reliability and vibro-acoustic characteristics were researched and confirmed by experimental studies.

We cannot ignore the active implementation of additive 3D-prototyping technologies in solving scientific and technical problems. This aspect has already firmly established itself today in the promoting of science field. With the help of such complexes (Figure 4), scientific and technological groups are able to quickly create models of the latest developments with the applied software packages use and 
implement them physically "in plastic" or "in metal" for carrying out experimental studies with the purpose of new solutions approbation in the energy sector. Of course, it significantly reduces the time spent on research and confirmation of the efficiency and feasibility of the new technologies use in power engineering.
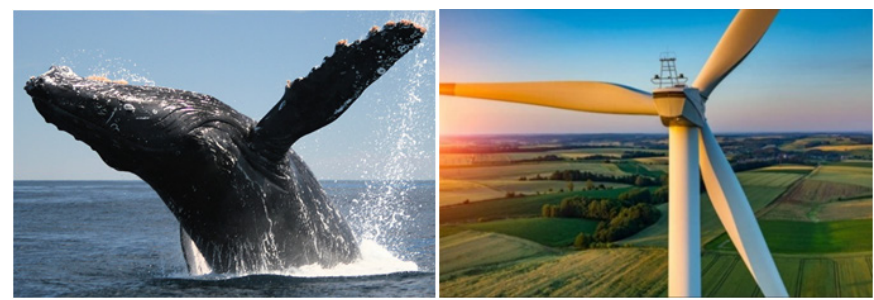

Figure I Use the whale humpback tubercles positive properties at the wind turbines.
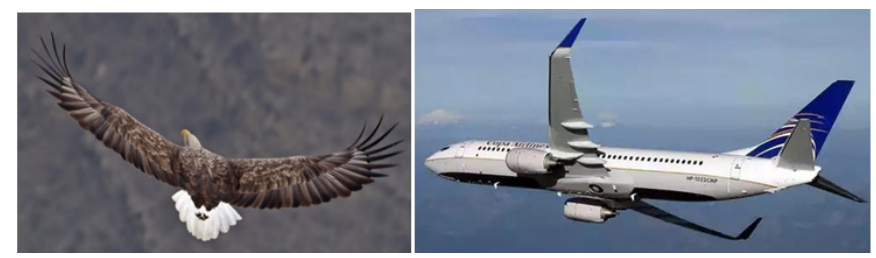

Figure 2 Use the ending eagle wings positive properties at the aviation.
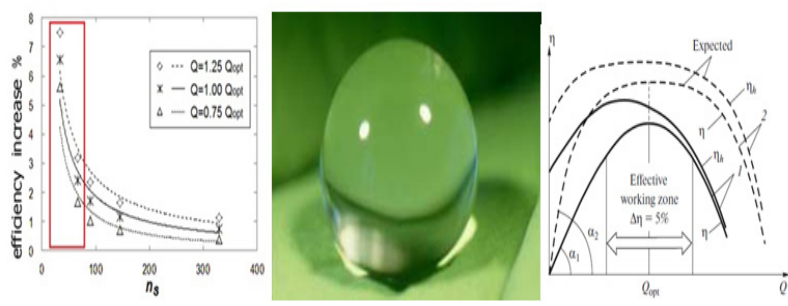

Figure 3 General view of depencies of total efficiency and hydraulic efficiency $\eta_{h}$ of the hydroturbine on capacity. Construction at the right

i. Traditional

ii. With a wide working area

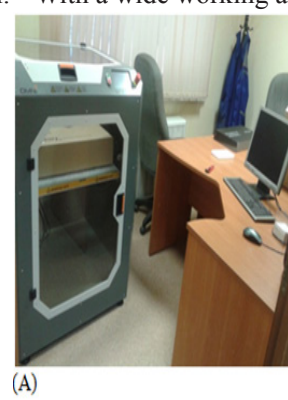

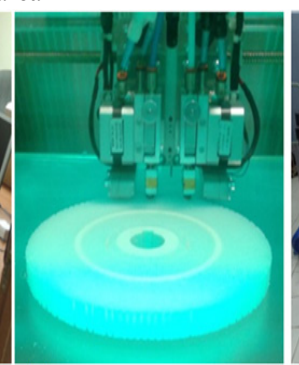

(B)

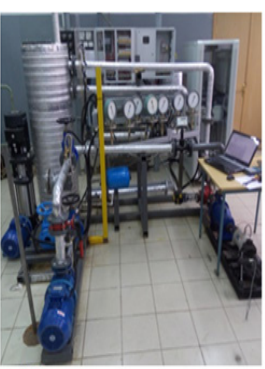

(C)
Figure 4 Research equipment NRU "MPEI".

A \& B) Complex of high-quality 3D-printing "OMNI 3D" with software for optimization of resource and time costs for manufacturing models

C) AN energy-cavitation bench for the complex study of the microhydroturbines characteristics.

From the point of view of alternative energy sources development it is worth noting an important world-scale scientific and technical direction. It is the designing of combined energy sources - a set of electric, heating and cooling supply in one device. Such complexes are convenient and effective primarily from the consumer point of view because they are energy intensive, simple and mobile power supply devices and are well suited to plug \& play technology.

Preliminary analytical studies in the combined energy supply field also show the urgent need to create efficient and reliable solutions that combining block-modular design, high density of assembly, use of RES and the microgeneration development based on them. Such long-term development trends are primarily justified due to the low prime cost of the energy complex and the rate value of its energy. In addition, due to the combined arrangement guaranteed energy efficiency about $10 \%$ is provided. ${ }^{3}$ Besides, today microgeneration is the main applicant for providing reliable and maneuverable energy supply when the power grid operates in high-risk modes, power failure (in case of ice rain, hurricane, etc.).

When moving in this direction of research, the energy efficient autonomous energy source based on micro HPP is developed and experimentally tested by the NRU "MPEI" scientific group. ${ }^{4}$ Its design consists of the build-in Kaplan turbine into the siphon penstock flow part (Figure 5). It fully meets the scientific and technical requirements that are indicated earlier and is guaranteed to provide:
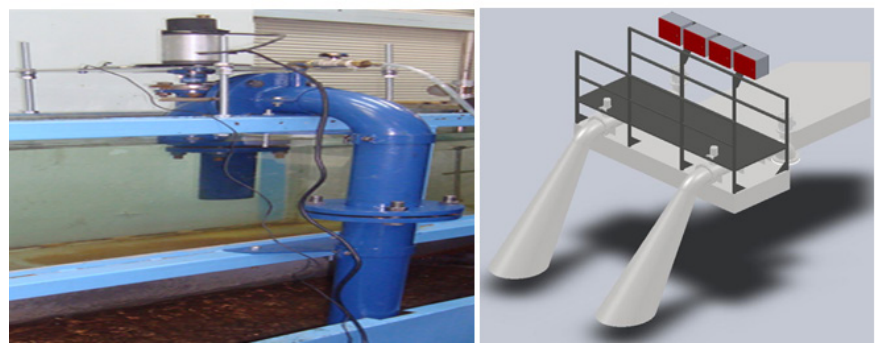

Figure 5 microHPP model with siphon penstock $(N=20 \mathrm{~W})$ at the NRU "MPEl" hydrodynamic watercourse and its natural-scale floating microHPP 3D-model $(\mathrm{N}=2 \times 1500 \mathrm{~W})$.

i. Compliance with modern requirements for environmental safety due to the use of environmentally friendly energy-intensive hydraulic resources

ii. Wide field of application in the real energy sector (in case of autonomous consumers power supply, production capacities of industrial and agricultural purposes energy supply, an energy source in the main pipeline cathodic protection systems ${ }^{5,6}$ as well as a way to restore small HPPs that decommissioned with preserved hydraulic engineering structures, etc.)

iii. The rate value reduction for generated energy due to the use of the energy source based on RES

iv. Shortening of costs due to increased reliability of energy supply

v. Hydraulic unit use with a high energy efficiency factor not less than $0,3849^{7}$ (allowing to reach the efficiency level not less than $90 \%$ ) over the existing level of small hydro turbines efficiency is $85 \%$

vi. The hydraulic unit high-efficiency electric drive use, which provides weight and dimensions improvement more than 1,5times, The electric drive efficiency increase is higher than the modern technical level at not less than $8 \%$ 
vii. The energy source design assembly features do not require significant costs for commissioning (in according to plug \& play technology) and provides installed capacity high maneuverability and mobility during transportation.

The economic effect of the energy source based on micro HPP was demonstrated using the estimate calculation example of medium-sized agricultural enterprise energy consumptions which engaged in cattle breeding and plant growing. Its energy consumption is estimated at $\sim 2$ millionkW*h/year. Even with a $50 \%$ transfer of the power supply of such an enterprise to the developed technology the saving energy costs will be $\sim 1880000$ RUR/year (more than $\$ 30000$ ).

The considered development directions of energy supply and energy saving are supported now by the Russian Federation Government at the legislative level by Strategy of scientific and technological development of the Russian Federation until 2035 year and Federal Scientific and Technical Program for the Development of Agriculture for 2017-2025 years.

Thus, improving the ways and approaches to design in modern researches is intended to supplement the scientific and technical foundations of the methodology for the creation of energy sources. Its realized using multiparametric optimization which takes into account the effects of biomimicry and regularities of the working bodies functional surfaces macrorelief influence on the working medium and the power equipment characteristics. Thereby to improve the environmental situation, increase the level of the world's population lives, and have a positive impact on the economy.

\section{Acknowledgements}

None.

\section{Conflict of interest}

The author declares no conflict of interest.

\section{References}

1. Miklosovic DS, Murray MM, Howle LE, et al. Leading-edge tubercles delay stall on humpback whale (Megaptera novaeangliae) flippers. Physics of Fluids. 2004;16(5):39-42.

2. Brüderlin $M$, Zimmer $M$, Hosters $N$, et al. Numerical simulation of vortex generators on a winglet control surface. Aerospace Science and Technology. 2017;71: 651-660.

3. Yaroslavov VA. Experience in construction of own generation facilities based on renewable energy sources. Report at International Conference "Own generation as a highly profitable business and a tool for a twofold reduction in energy costs". Crocus Expo, Moscow, Russia; 2017.

4. Volkov AV, Druzhinin AA. About the question of designing the floating lowhead micro HPP. Youth scientific and technical bulletin №9. Moscow, Russia: Bauman Moscow State Technical University; 2015.

5. Parygin AG, Volkov AV, Ryzhenkov AV, et al. About the opportunities the application of the low head micro hydraulic power plants for the autonomous power supply of the technological equipment of the oil pipelines. Moscow, Russia: Neftyanoe Khozyaystvo-Oil Industry; 2015. p. $127-130$.

6. Alexander G Parygin, Alexander V Volkov, Artyom V Ryzhenkov, et al. On possibilities of creating systems for autonomous power supply of pipeline cathodic protection stations. RJPBCS. 2016;7(6):561-569.

7. Parygin AG, Volkov AV, Ryzhenkov AV, et al. Optimization algorithm of parameters of low head microhydropower plant at an early design stage. International Journal of Applied Engineering Research. 2016;11(22):10878-10886 